\title{
Congruence and metaplectic covariance: rational biquadratic reciprocity and quantum entanglement
}

\author{
WALTER J. SCHEMPP*
}

\begin{abstract}
The purpose of the paper is to elucidate the cyclotomographic applications of the coadjoint orbit methodology to the Legendre-Hilbert-Artin symbolic tower of class field theory in the sense of the number field theories of Chevalley, Hasse, Weil and Witt. The Witt arithmetics concludes with the law of rational biquadratic reciprocity and quantum entanglement.
\end{abstract}

Keywords: Third order principle of spinor triality, spaces of even and odd half-spinors, Hopf principal circle bundle, metaplectic Lie group $\mathrm{Mp}(2, \mathbb{R})$, the metaplectic coadjoint orbit model and spherical contact geometry, the first maxim of the geometric quantization principle, half-spinor Maslov index, Witt quartic groups, cyclotomography, LegendreHilbert-Artin symbolic tower of class field theory, valuation and module function, adéles and idéles, differential idéles, rational quantum entanglement, pure spinor invariants of octonion geometry, magnetic resonance tomography and angiography, symplectic spectroscopy at molecular level.

2020 Mathematics Subject Classification: 11R18, 11S25, 11T22.

Dedicated to Professor Francesco Altomare, on occasion of his 70th birthday, with esteem and friendship.

\section{INTRODUCTION}

The law of quadratic reciprocity is one of the cornerstones of the classical theory of numbers. The theorem on primes in arithmetic progressions was a key ingredient in Adrien-Marie Legendre's (1752-1833) attempted proof of the law of quadratic reciprocity. He coined the term "reciprocity". The most penetrating classical approach of quadratic reciprocity which is suggestive of substantial generalizations is due to Carl Friedrich Gauß (1777-1855). His very first proof of the law of quadratic reciprocity by means of a remarkable induction argument over the primes was published in the treatise Disquisitiones Arithmeticx of 1801. The concept of Gaussian sum for quadratic forms has included the laws of quadratic and biquadratic reciprocity into the field of constructive mathematical analysis.

In a letter to the astronomer Heinrich Wilhelm Matthias Olbers, Gauß wrote in 1805:

"This lack of sign has overcast everything else I have found, and since four years hardly a week has passed in which I did not make an attempt to no avail of resolving this knot. But all the brooding, all the searching was in vain, and each time I was forced to put down the pen in sorrow. Finally, a few days ago, I was successful - but not by my arduous search but only by the grace of God, as I would say. Like lightening strikes the riddle was solved; I myself would be unable to tell you the connection between what I knew before, in my last attempts - and the idea by which I finally succeeded. Curiously the solution of the problem now appears to be easier than many other results which have not cost me as

Received: 03.10.2020; Accepted: 16.11.2020; Published Online: 01.03.2021

*Corresponding author: Walter J. Schempp; schempp@mathematik.uni-siegen.de

DOI: $10.33205 / \mathrm{cma} .804852$ 
many days as the present problem cost me years, and certainly no one will get any idea about the tight squeeze of feeling which besieged me for so a long time when I eventually present this matter."

The original form of the law of quadratic reciprocity that Gauß proved reads

$$
\left(\frac{p}{q}\right)=\left(\frac{(-1)^{\frac{q-1}{2}} q}{p}\right)
$$

for any distinct odd primes $\{p, q\}$. This equation makes already the quantum entanglement phenomenon apparent. However Gauß never explicitly employed the concept of group, but many group theoretical results are found in the treatise Démonstration de quelques théorèmes concernants les périodes des classes de formes binaires du second degré of 1876 . The concept of symplectic Lie group $\operatorname{Sp}(2, \mathbb{R}) \cong \mathrm{SL}(2, \mathbb{R})$, its two-fold covering group which is formed by the metaplectic Lie group

$$
\mathrm{Mp}(2, \mathbb{R}) \hookrightarrow \mathrm{U}\left(L^{2}(\mathbb{R}), \mathbb{C}\right)
$$

and its strong operator action as a deus ex machina of the analytic theory of quadratic forms remained outside his way of mathematical reasoning. This has been done later on by the investigations of Carl Ludwig Siegel (1896-1981) ([39], [21]). The present paper offers a Galois cohomology approach to the metaplectic Schaar-Landsberg construction. It can be considered as an outgrowth of local class field theory and the third order principle of spinor triality which emphasizes impressively the extraordinary role of the finite place 2 of the prime field $\mathbb{Q}$ of rational numbers corresponding to the embedding $\mathbb{Q} \hookrightarrow \mathbb{Q}_{2}$ into the quasifactor $\mathbb{Q}_{v}$ of the topological ring of adéles $\mathbb{Q}_{\mathbb{A}}$ belonging to the associated 2-adic valuation $v$ ([8], [38]).

The motivation of Gauß in seeking new proofs of the law of quadratic reciprocity was to develop methods for treating higher reciprocity laws. This can be read off the treatise Theorematis fundamentalis in doctrina de residuis quadraticis demonstrationes et amplicationes novæ of 1817 ([7]). The metaplectic group realization

$$
\operatorname{Mp}(4, \mathbb{R}) \hookrightarrow \mathrm{U}\left(L^{2}(\mathbb{R} \oplus \mathbb{R}), \mathbb{C}\right)
$$

suggests an extension to the law of biquadratic reciprocity on $\mathbb{C} \oplus \mathbb{C} \hookrightarrow \mathbb{O}$. In his second memoir on biquadratic residues, Gauß stated, without proof, the law of biquadratic reciprocity in 1832. Subsequently, Ferdinand Gotthold Max Eisenstein (1823-1852) published several proofs of the mysteries of the higher arithmetic in 1844.

The merit of the approach of Gauß and Eisenstein is that they pointed out the way of the laws of reciprocity of classical number theory to Artin's law of reciprocity and the duality determined by Hilbert's symbol. Hasse's law of reciprocity is in close connection with Artin's law. Actually, Helmut Hasse (1898-1979) opened the road jointly with Hensel to the work of Chevalley and Artin.

\section{THE LEGENDRE-HILBERT-ARTIN SYMBOLIC TOWER}

The ability to detect ultra-precisely the interaction of light and matter at the single-particle level represents a particular important application of the formalism of local class field theory to quantum optics and quantum information processing. In terms of class field theory over the local fields $\mathbb{R}$ and $\mathbb{C}$ of characteristic zero, ultra-precise ion clocks represent a type of factor-sets which are attached to the cyclic Galois extension of degree 2 of the groundfield

$$
\mathbb{R} \cong \mathbb{Q}_{\infty} .
$$




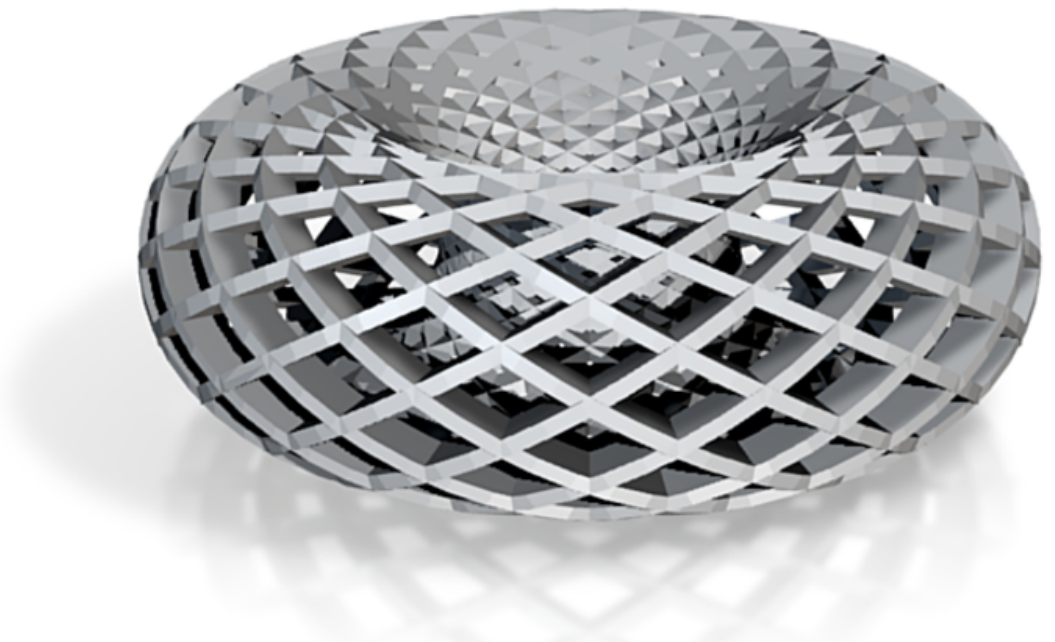

FIGURE 1. Cyclotomographic visualization of a two-fold covering of the twodimensional torus $\mathbb{T}^{2}$. The leaves of the foliation define pairs of gradient controllable Villarceau circles. The fundamental group is $\pi_{1}\left(\mathbb{T}^{2}\right)=\mathbb{Z} \oplus \mathbb{Z} \hookrightarrow$ $\mathrm{WQ}\left(\mathbb{Q}_{2}\right)$ to implement the law of rational biquadratic reciprocity by the spinor triality and the action of the Witt quartic group $\mathrm{WQ}\left(\mathbb{Q}_{2}\right) \cong \mathbb{Z}_{2} \times \mathbb{Z}_{8} \times \mathbb{Z}_{2}$.

According to Galois cohomology, it is sufficient to use a quantum entangled state to amplify the momentum an ion receives upon scattering a photon. In this vein, the following result of Galois cohomological quantum metrology arises:

Theorem 2.1. Every equivalence class of simple central associative algebras over the groundfield $\mathbb{Q}_{\infty} \cong$ $\mathbb{R}$ contains a cyclic algebra $[\mathbb{C} \mid \mathbb{R} ;\{\chi, \theta\}]$ of non-trivial character $\chi$ of the Galois group $\mathcal{G}$ of $\mathbb{C}$ over $\mathbb{R}$ given by $\chi(\varsigma)=e^{\pi i}=-1$, and the coboundary factor $\theta \in \mathbb{R}^{\times}$of quantum entanglement so that the mapping $\theta \rightsquigarrow\{\chi, \theta\}$ is a morphism of $\mathbb{R}^{\times}$into the group $\mathrm{H}(\mathbb{R})$ of cyclic factor-classes of $\mathbb{R}$ with kernel formed by the group of norms of $C^{\times}$of the cyclic Galois extension $C=\mathbb{C} \mid \mathbb{R}$ of ramification index 2 over $\mathbb{R}$ attached to the character $\chi \in \widehat{\mathcal{G}}$ of second order, and the coboundary factor $\theta$ which corresponds to the four-fold half-spinor Maslov index.

The only non-trivial cyclic algebra over the groundfield $\mathbb{R} \cong \mathbb{Q}_{\infty}$ of this kind is the fourdimensional real division algebra of classical quaternions

$$
\mathbb{H} \cong \mathbb{C} \otimes_{\mathbb{R}} \mathbb{C} \cong[\mathbb{C} \mid \mathbb{R} ;\{\chi,-1\}]
$$

with non-trivial character $\chi \in \widehat{\mathcal{G}}$ of second order, and non-trivial coboundary factor $\theta=-1 \in \mathbb{R}_{-}^{\times}$induced by reflection of the norm image $N_{\mathbb{C} \mid \mathbb{R}}\left(\mathbb{C}^{\times}\right)=\mathbb{R}_{+}^{\times}$which is a subgroup of $\mathbb{R}^{\times}$of index 2 . Corresponding to the Pauli spin matrices in $\mathrm{SU}(2, \mathbb{C})$, the vectors of the canonical 
basis of $\mathbb{H}$ satisfy the same relations as the matrices

$$
\left\{\left(\begin{array}{cc}
+1 & 0 \\
0 & +1
\end{array}\right),\left(\begin{array}{cc}
+1 & 0 \\
0 & -1
\end{array}\right),\left(\begin{array}{cc}
0 & +1 \\
+1 & 0
\end{array}\right),\left(\begin{array}{cc}
0 & +1 \\
-1 & 0
\end{array}\right)\right\} .
$$

With exception of the matrix of the identity transformation with trace 2 , the other matrices are traceless. The last matrix of the quadruple is the symplectic matrix

$$
-J=\left(\begin{array}{cc}
0 & +1 \\
-1 & 0
\end{array}\right)
$$

with $J^{-1}={ }^{t} J=-J \in \mathrm{Sp}(2, \mathbb{R}) \cong \mathrm{SL}(2, \mathbb{R})$. Referring to the nomenclature of the Steinberg symbolic calculus ([29], [30]), the real division algebra $\mathbb{H}$ takes the form

$$
(+1,+1)_{\mathbb{R}} \cong(+1,-1)_{\mathbb{R}} \cong(-1,-1)_{\mathbb{R}} \cong(-1,+1)_{\mathbb{R}}
$$

provided the symbols on right hand side represent a skew field, or equivalently, their multiplicative norm form is non-isotropic and therefore hyperbolic. In particular, the symbolic calculus involves the isomorphies

$$
(a, b)_{\mathbb{R}} \cong\left(a a^{\prime 2}, b b^{\prime 2}\right)_{\mathbb{R}}
$$

for $\left\{a, a^{\prime}, b, b^{\prime}\right\} \subset \mathbb{R}^{\times}$.

In view of the third order principle of spinor triality, it is a remarkable observation that the non-zero pure quaternions in $\mathbb{H} \hookrightarrow \mathbb{O}$ are characterized by the fact that they are not belonging to the groundfield $\mathbb{R} \cong \mathbb{Q}_{\infty}$, but their squares are. Thus, the three-dimensional vector subspace of $\mathbb{H}$ consisting of the pure quaternions takes the form

$$
\left\{q \in(a, b)_{\mathbb{R}} \mid q^{2} \in \mathbb{R}, q \notin \mathbb{R}^{\times}\right\} .
$$

In accordance with the theorem of Alexander Merkurjev concerning the quaternion symbol ([23], [32]), the central simple algebra $\mathbb{H}$ gives rise to the:

Corollary 2.1. The coboundary factor $\theta \in \mathbb{R}^{\times}$of quantum entanglement attached to the quaternion quantization procedure is given by the quaternion symbol

$$
\left(\bullet, \bullet^{\prime}\right)_{\mathbb{R}}: \mathbb{R}^{\times} \times \mathbb{R}^{\times} \longrightarrow \operatorname{Br}(\mathbb{R}) \cong \mathbb{Z}_{2} \cong \frac{1}{2} \mathbb{Z} / \mathbb{Z} \hookrightarrow \mathbb{Q} / \mathbb{Z} .
$$

It is universal in the sense that every other quaternion symbol can be factored over it in the third Galois cohomology $\mathrm{H}^{3}\left(\mathbb{R}, \mathbb{Z}_{2}\right)$ of the third order principle of spinor triality.

A standard functorial argument establishes the fact that a universal symbol is uniquely determined up to a unique isomorphism. Since the half-spinor norm form of the metaplectic coadjoint orbit model determines completely its quaternion algebra, the following result arises in the rational case:

Theorem 2.2. Up to an isometry the quaternion symbol $(-1 .-1)_{\mathbb{Q}_{2}}$ defines the only non-split quaternion algebra over the commutative field of 2-adic rational numbers $\mathbb{Q}_{2}$.

Notice that the quasifactor $\mathbb{Q}_{2}$ of the adélic ring $\mathbb{Q}_{\mathbb{A}}$ is either the completion $\mathbb{Q}_{v}$ of the prime field $\mathbb{Q}$ of rational numbers with respect to the ultrametric distance associated to the 2-adic valuation

$$
v=|\bullet|_{2}
$$

or $\mathbb{Q}_{v}$ denotes for $v=\infty$ the completion $\mathbb{Q}_{\infty}=\mathbb{R}$. Of course, the topology associated to the ultrametric distance is locally compact and not discrete. The closure $\mathbb{Z}_{2}$ of the rational integers $\mathbb{Z} \hookrightarrow \mathbb{Q}$ in $\mathbb{Q}_{2}$ defines a compact neighborhood of 0 in $\mathbb{Q}_{2}$. The algebraic theory of quadratic 
forms originated in a seminal paper of 1937 by Ernst Witt (1911-1991) ([30], [32]). The Witt quartic group $\mathrm{WQ}\left(\mathbb{Q}_{2}\right)$ will help to detect the pure half-spinors acting by rational quantum entanglement according to the third order principle of spinor triality.

\section{THE SYMPLECTIC SPINOR PAIR OF GROUPS $(\operatorname{Mp}(2, \mathbb{R}), \operatorname{Br}(\mathbb{R}))$}

Let $\widehat{\mathcal{N}}$ denote the unitary dual of the (2+1)-dimensional real unipotent Heisenberg Lie group $\mathcal{N}$ consisting of the equivalence classes of irreducible unitary linear representations of $\mathcal{N}$ in complex Hilbert spaces ([15]). The geometric model of $\widehat{\mathcal{N}}$ derives in spherical contact geometry from the first maxim of the geometric quantization principle:

"Never look at the orbits of the adjoint action - rather always look at the orbits of the coadjoint action".

Actually, it is the quantization maxim which permits the transition from number theory to symplectic spectroscopy at molecular level in terms of the third order principle of spinor triality.

Let the subgroup $G \hookrightarrow \mathrm{U}\left(L^{2}(\mathbb{R}), \mathbb{C}\right)$ be the covariance group associated with $\widehat{\mathcal{N}}$. Then, the exact sequence

$$
\{1\} \longrightarrow \mathbb{T} \longrightarrow G \stackrel{\nu}{\longrightarrow} \mathrm{Sp}(2, \mathbb{R}) \longrightarrow\{1\}
$$

arises, where $\mathbb{T}=\mathbb{R} / \mathbb{Z} \cong \mathrm{U}(1, \mathbb{C})$ denotes the one-dimensional compact torus group. The mapping $\nu: G \longrightarrow \mathrm{Sp}(2, \mathbb{R})$ is a Lie group homomorphism and the differential $\nu_{*}$ a real Lie algebra isomorphism. Concerning the inverse Fourier transforms $\mathcal{F}_{\mathbb{R}}$ and $\overline{\mathcal{F}}_{\mathbb{R}}$, it is important to note the spin echo projections

$$
\nu\left(\mathcal{F}_{\mathbb{R}}\right)=J, \quad \nu\left(\overline{\mathcal{F}}_{\mathbb{R}}\right)=J^{-1} .
$$

The restriction $\mu=\nu \mid \mathrm{Mp}(2, \mathbb{R})$ defines the exact sequence

$$
\{1\} \longrightarrow \mathbb{Z}_{2} \longrightarrow \mathrm{Mp}(2, \mathbb{R}) \stackrel{\mu}{\longrightarrow} \mathrm{Sp}(2, \mathbb{R}) \longrightarrow\{1\} .
$$

Taking into account the half-spinor Maslov index $-\frac{1}{2}$ of $\operatorname{Mp}(2, \mathbb{R})$, the 2-cocycle $e^{-\frac{\pi i}{4}}$ of $\mathrm{Sp}(2, \mathbb{R})$, the bijective differential $\mu_{*}$ of $\mu: \mathrm{Mp}(2, \mathbb{R}) \longrightarrow \mathrm{Sp}(2, \mathbb{R})$ and the symplectic spinor configuration inside the real dual vector space $\mathfrak{L i e}(\mathcal{N})^{*}$ of the real nilpotent Heisenberg Lie algebra $\mathfrak{L i e}(\mathcal{N})$, the universal quaternion symbol $\left(\bullet, \bullet^{\prime}\right) \mathbb{R}$ affords by character composition of the entangled ingredients the metaplectic Schaar-Landsberg construction of the third Galois cohomology $\mathrm{H}^{3}\left(\mathbb{R}, \mathbb{Z}_{2}\right)$ of the third order principle of spinor triality ([5], [8]).

Theorem 3.3. For any integral numbers $p$ and $q \geq 1$, the metaplectically entangled Schaar-Landsberg identity

$$
\frac{e^{-\frac{\pi i}{8}}}{\sqrt{p}} \sum_{0 \leq m \leq p-1} e^{2 \pi i m^{2} \frac{q}{p}}=\frac{e^{\frac{\pi i}{8}}}{\sqrt{2 q}} \sum_{0 \leq n \leq 2 q-1} e^{-\frac{\pi i n^{2}}{2} \frac{p}{q}}
$$

holds. The opposite $22.5^{\circ}$ phase factors on both sides of the equality derive from the Galois cohomological meaning of the 2-cocycle as an octonionic half-spinor root of unity. The action of the symplectic spinor can be visualized by the Picard tori of the Hopf principal circle bundle.

The metaplectically entangled Schaar-Landsberg identity was first discovered in 1850 by Mathieu Schaar (1817-1867) who proved it using the Poisson summation formula, and proceeded to derive from it the law of quadratic reciprocity. In 1893, Georg Landsberg (1865-1912), 


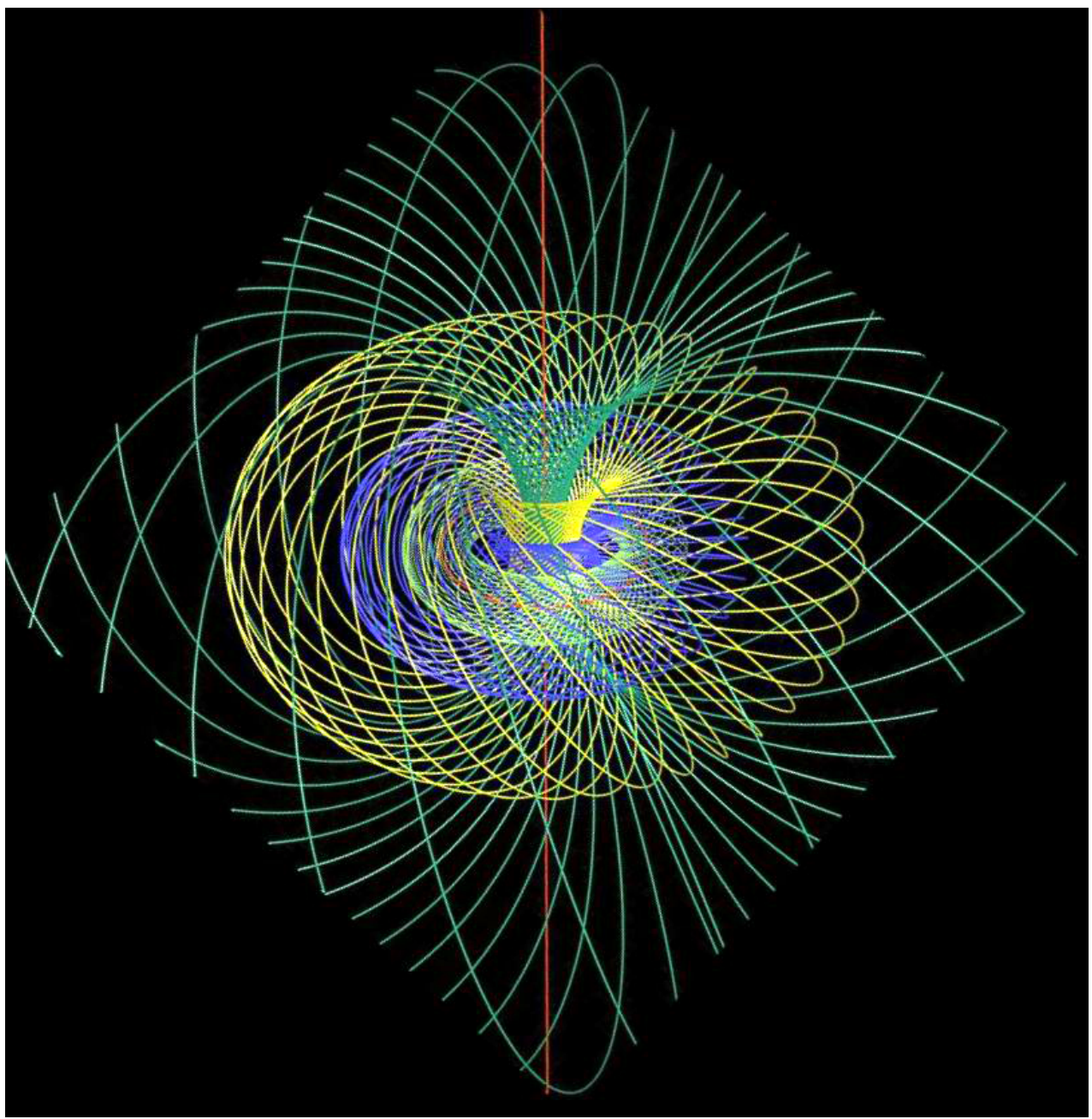

FIGURE 2. Cyclotomography of the Riemann-Roch structure on the torus $\mathbb{T}^{2}$ : The Hopf principal circle bundle visualizing the cyclic factor-sets of the first Galois cohomology $\mathrm{H}^{1}(\mathbb{R}, \operatorname{Spin}(3, \mathbb{R}))$ in the context of the third cohomology $\mathrm{H}^{3}\left(\mathbb{R}, \mathbb{Z}_{2}\right)$ which represents the third order principle of spinor triality and implements the law of quadratic reciprocity. In terms of the linear theory of theta functions with $\mathbb{T}$-valued quadratic characters, the Hopf fibration receives its Riemann-Roch structure on tori from the cyclic extension of the Poisson summation formula to the adélic ring of the field $\mathbb{R}$. For the period sublattice $\Lambda_{1} \hookrightarrow$ $\mathbb{C}$, the coordinatization by the affine $\mathbb{C}$-basis $\left\{1, \wp_{\Lambda_{1}}, \wp_{\Lambda_{1}}^{\prime}\right\}$ defines a projective embedding of the two-dimensional Picard torus $\left(\bmod \Lambda_{1}\right)$ by a non-singular elliptic curve $E(\mathbb{C})$ in the complex projective plane $\mathbb{P}_{2}(\mathbb{C}) \hookrightarrow \mathbb{P}_{\mathbb{C}}\left(\mathfrak{L} \mathfrak{i}(\mathcal{N})^{*}\right)$ inside the projectivized dual of the real nilpotent Heisenberg Lie algebra $\mathfrak{L} \mathfrak{i}(\mathcal{N})$. 
who was the coauthor of Hensel's treatise on algebraic functions of one variable and was unaware of Schaar's work, rediscovered a slightly more general version of the relation. The arithmetician Kurt Hensel (1861-1941) created the $p$-adic methodology which places the theory of quadratic reciprocity in the natural frame of modern number theory ([40]). Then, its adélic language permits to unify the various techniques of significantly stepping forward. In Section 6 infra, an interpretation of the metaplectically entangled Schaar-Landsberg construction will be given in terms of theta differential idéles of module 1.

The evaluation of the basic Gaussian quadratic sum is performed by putting $q=1$ :

$$
\sum_{0 \leq n \leq p-1} e^{\frac{2 \pi i n^{2}}{p}}=\sqrt{\frac{p}{2}} e^{\frac{\pi i}{4}}\left(1+e^{-\frac{\pi i}{2}}\right)=\sqrt{p} i^{\left(\frac{p-1}{2}\right)^{2}} .
$$

Due to the congruence modulo 4

$$
\left(\frac{p q-1}{2}\right)^{2}-\left(\frac{p-1}{2}\right)^{2}-\left(\frac{q-1}{2}\right)^{2}=\frac{(p-1)(q-1)}{2},
$$

the law of quadratic reciprocity for any distinct odd primes $\{p, q\}$ takes the standard form in terms of the Legendre quadratic symbol

$$
\left(\frac{p}{q}\right)=\left(\frac{p}{q}\right)_{2}=\left\{\begin{array}{rll}
1 & : & p \text { is a quadratic residue } \bmod q \\
-1 & : & p \text { is a quadratic nonresidue } \bmod q
\end{array}\right.
$$

over the field $\mathbb{Q}$ of rational numbers

$$
\left(\frac{p}{q}\right)\left(\frac{q}{p}\right)=(-1)^{\frac{(p-1)(q-1)}{4}}
$$

as the product of multiplicative characters of $\mathbb{Z}_{p}^{\times}$and $\mathbb{Z}_{q}^{\times}$, respectively. The Legendre quadratic symbol $(\bullet)=\left(\bullet \bullet_{\bullet^{\prime}}\right)_{2}$, invariably so written by most classical authors is defined whenever the lower variable is a prime and the upper variable an integer prime to the lower variable, which admits the value +1 when the upper variable is a quadratic residue modulo the lower variable, and - 1 otherwise ([2]). The quantum entangled Schaar-Landsberg identity can also used to establish the so-called supplementary theorem which includes the first supplement

$$
\left(\frac{-1}{p}\right)=(-1)^{\frac{p-1}{2}}
$$

and the second supplement

$$
\left(\frac{+2}{p}\right)=(-1)^{\frac{p^{2}-1}{8}}
$$

on the quadratic residue behaviour of -1 and +2 , respectively. The multiplicativity of the Legendre quadratic symbol implies the equation

$$
\left(\frac{-1}{q}\right)^{\frac{p-1}{2}}\left(\frac{p}{q}\right)=\left(\frac{q}{p}\right)
$$

so that the first supplement affords the law of quadratic reciprocity. Tensoring rational quaternion algebras over the field of $p$-adic numbers $\mathbb{Q}_{p}$ including $\mathbb{Q}_{\infty} \cong \mathbb{R}$ provides by means of the quaternion symbol $\left(\bullet, \bullet^{\prime}\right)_{\mathbb{Z}}$ the Hilbert reciprocity law which generalizes the quadratic reciprocity law. The global reciprocity law which relies on normic aspects of class field theory forms a monumental generalization of the classical law of quadratic reciprocity. 
In terms of class field theory, the classical quadratic reciprocity law is equivalent to the assertion that an extension of the field of rational numbers $\mathbb{Q}$ admits a Hecke L-function. Over the years, however, the L-functions have been expunged from class field theory and have been replaced by an algebraic edifice based on Galois cohomology. The avoidance of the concepts of cohomology theory gives rise to an artificial flair. In the context of harmonic analysis on Abelian groups, the universal quaternion symbol $\left(\bullet, \bullet^{\prime}\right)_{\mathbb{R}}$ allows to circumvent the standard reference of the famous functional equation for Jacobi's theta function as a special case of the Poisson summation formula and its cyclic Eisenstein extension ([38]).

Remark 3.1. The induction arguments of Gauß to prove the Theorema Aureum are closely related to the calculations of the Witt quartic group isomorphisms by splitting off metabolic subspaces ([32]). Using the conventions of basic number theory ([38], [32]), the Witt geometric method yields

$$
\mathrm{WQ}\left(\mathbb{F}_{2}\right) \cong \mathbb{Z}_{2}, \quad \mathrm{WQ}(2) \cong \mathbb{Z}_{2} \oplus \mathbb{Z}_{8}, \quad \mathrm{WQ}\left(\mathbb{Q}_{2}\right) \cong \mathbb{Z}_{2} \times \mathbb{Z}_{8} \times \mathbb{Z}_{2}
$$

in terms of the half-spinor Maslov index associated as an octonionic root of unity

$$
e^{\frac{\pi i}{4}}=\frac{1+e^{\frac{\pi i}{2}}}{\sqrt{2}}=\frac{1+i}{\sqrt{2}}
$$

with the generic coadjoint orbit $\mathcal{O}_{1} \in \mathfrak{L} \mathfrak{i}(\mathcal{N})^{*} / \operatorname{CoAd}(\mathcal{N})$. Thus, the Witt quartic groups $\operatorname{WQ}(2) \hookrightarrow$ $\mathrm{WQ}\left(\mathbb{Q}_{2}\right)$ are adapted to the third order principle of spinor triality by the projection onto the direct factor relative to the direct product representation

$$
\mathrm{WQ}(\mathbb{Q}) \longrightarrow \mathrm{WQ}\left(\mathbb{Q}_{2}\right) \longrightarrow \mathbb{Z}_{8}
$$

which actually is a Gaussian sum. The invariant of the order $2^{3}=\operatorname{dim}_{\mathbb{R}} \mathbb{O}$ of the alternative and biassociative division $\mathbb{R}$-algebra of octonions $\mathbb{O}$ on the Cayley-Dickson scale

$$
\mathbb{R} \hookrightarrow \mathbb{C} \hookrightarrow \mathbb{H} \hookrightarrow \mathbb{O}
$$

with continuous module function $\bmod _{\mathbb{R}}$ of respective Haar measures

$$
|\bullet|,|\bullet|^{2},\left|\bullet \bullet^{\prime \prime}\right|^{4},\left|\bullet^{\prime \prime \prime}\right|^{8}
$$

is due to Johann Peter Gustav Lejeune Dirichlet (1805-1859) ([31], [35]). Moreover, the module function of the 2-adic case affords the identity

$$
\bmod _{\mathbb{Q}_{2}}(2)=\frac{1}{2}
$$

which defines a $\mathrm{WQ}\left(\mathbb{Q}_{2}\right)$-invariant model of the plane coadjoint orbit $\mathcal{O}_{\frac{1}{2}} \in \mathfrak{L i e}(\mathcal{N})^{*} / \operatorname{CoAd}(\mathcal{N})$ in concordance with Theorem 1 supra. Due to the third order principle of spinor triality, the coadjoint orbit model $\mathfrak{L} \mathfrak{i}(\mathcal{N})^{*} / \operatorname{CoAd}(\mathcal{N})$ of the unitary dual $\widehat{\mathcal{N}}$ of the $(2+1)$-dimensional real unipotent Heisenberg Lie group $\mathcal{N}$ implements the non-invasive imaging modality of gradient controlled clinical magnetic resonance tomography and angiography of radiological diagnostics in current clinical use ([1], [4], [33]). To include dynamical phenomena into the spherical contact geometry of $\mathfrak{L i \mathfrak { e }}(\mathcal{N}) * / \operatorname{CoAd}(\mathcal{N})$, functional magnetic resonance permits the modalities of diffusion and perfusion magnetic resonance imaging ([18], [19], [25], [36], [37]).

Just as in the earlier studies of Legendre, Gauß distinguishes eight separate cases according to the different nature of the individual primes in order to present a natural crystallization of the special cases that had been discovered earlier by Leonhard Euler (1707-1783). It was Dirichlet's modification of the Gaussian proof which completed in 1854 the induction procedure over the upper and lower sequences of prime variables $\{P, Q\}$ that are enumerated by the 


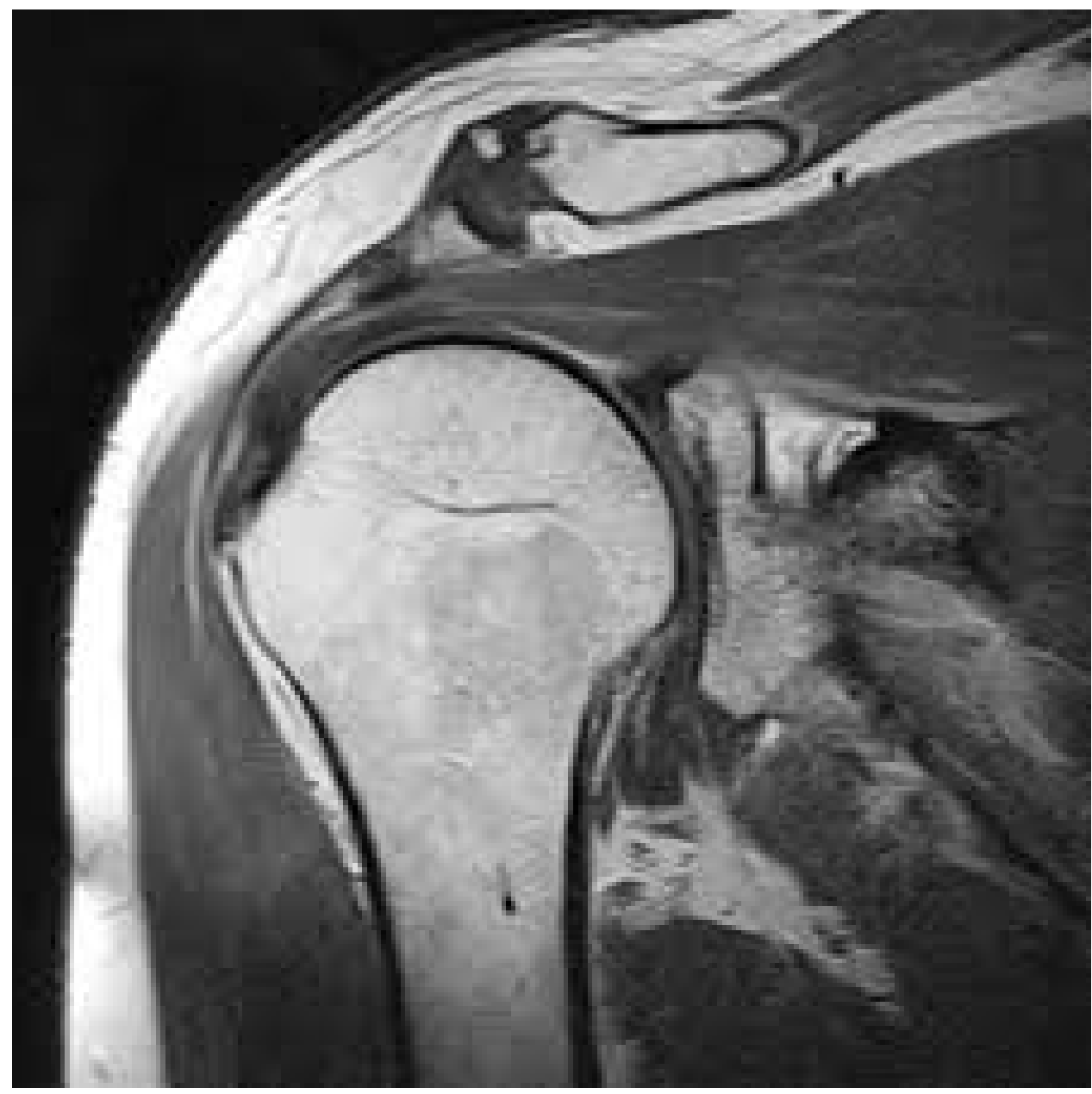

FIGURE 3. Gradient controlled clinical magnetic resonance tomography: Non-invasive image of the musculoskeletal anatomy of a normal right shoulder joint (Articulatio glenohumeralis). The supraspinatus, infraspinatus and teres minor muscles and tendons are shown. They all attach to the greater tuberosity. The tendons and rotator cuff muscles act to stabilize the shoulder joint during movements.

Witt quartic group $\mathrm{WQ}(\mathbb{Z}) \cong \mathrm{WQ}(\mathbb{R}) \cong \mathbb{Z}$ in the idélic product

$$
\left(\frac{P}{Q}\right)\left(\frac{Q}{P}\right)=\prod_{\{p, q\}}\left(\frac{p}{q}\right)\left(\frac{q}{p}\right) .
$$

Finally, the inductive conclusion over the primes reads

$$
\left(\frac{P}{Q}\right)\left(\frac{Q}{P}\right)=(-1)^{\frac{(P-1)(Q-1)}{4}}
$$

as desired ([2], [11]). The seventh proof of Gauß is based on cyclotomy which anticipates the idélic procedure of pasting together local cyclic data into a global object. Originally these global 


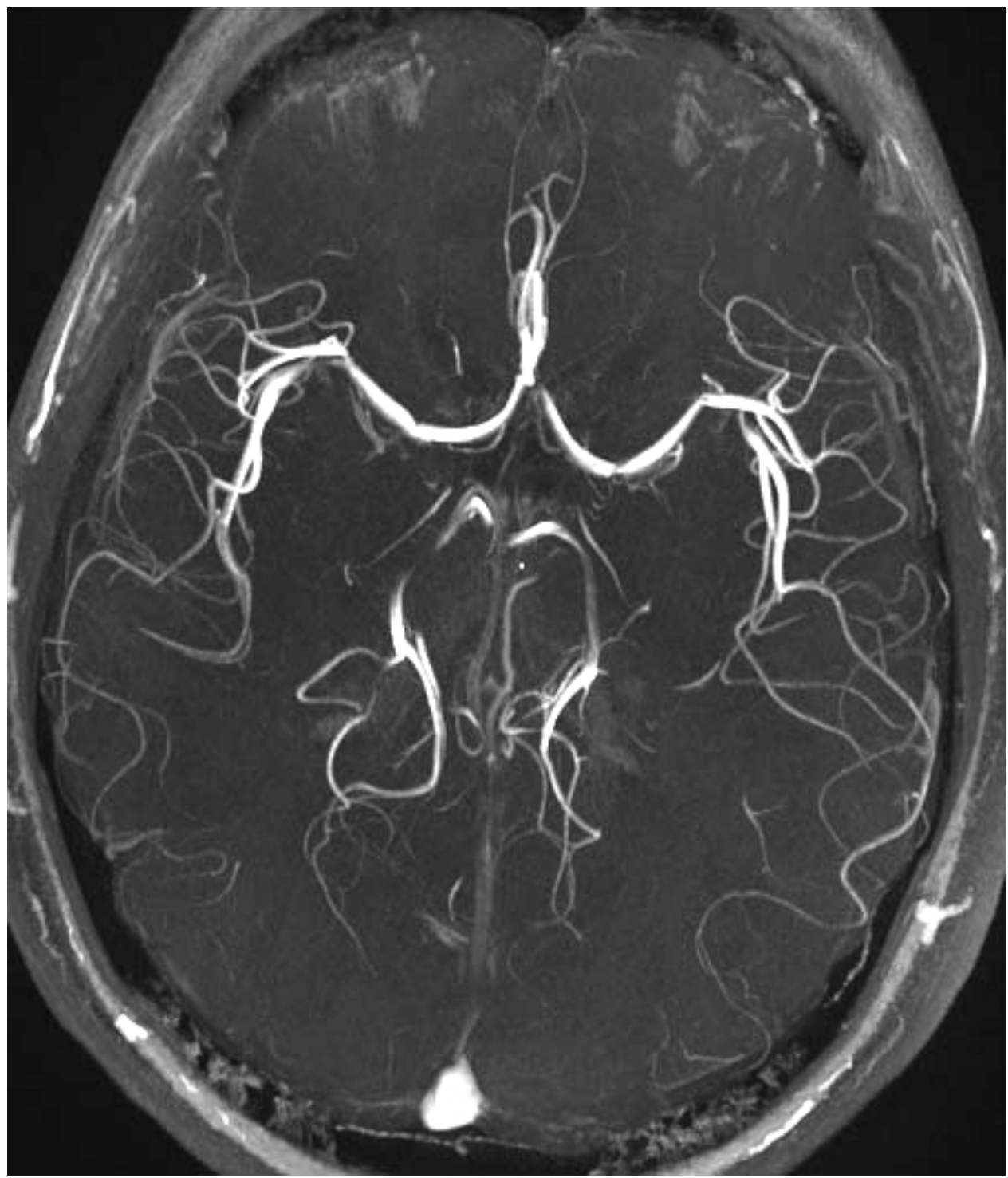

FIGURE 4. Gradient controlled clinical magnetic resonance angiography: Intercranial time-of-flight non-invasively performed on a healthy male adult acquired at a 3.0 Tesla magnetic field density. Maximum intensity projection in the axial direction demonstrates excellent blood to background contrast and depiction of small peripheral intercranial vessels. The high resolution of the angiogram can be enhanced by electrocardiogram gating and the administratio of a contrast agent. Higher static magnetic field density permits to increase the spatial resolution in time-of-flight and the temporal resolution in dynamic magnetic resonance angiography. 
objects were known as ideal elements or idéles. In modern terms partly due to the seminal work of Chevalley and Weil, the cyclotomic extension to cyclotomography combines the factor-sets of quadratic periods of the cylotomographic equation

$$
\frac{X^{p-1}-1}{X-1}=0
$$

for positive odd primes $p$ with cyclic Galois extensions. Gauß's seventh proof, which has been admired by Dirichlet, introduces theta type data of a Frobenius decomposition of quadratic periods by means of the inverse affine linear tomographic gradient mapping

$$
X \rightsquigarrow 2 X+1=Y
$$

of metaplectically invariant quadratic periods within the generic coadjoint orbit $\mathcal{O}_{1} \in \mathfrak{L} \mathfrak{i}(\mathcal{N}) * / \operatorname{CoAd}(\mathcal{N}$ ([21]). The discriminant-congruence $\bmod q$ of the cylotomographic equation transforms into the solvability condition in original form of Gauß

$$
\left(\frac{(-1)^{\frac{p-1}{2}} p}{q}\right)=+1
$$

for positive odd primes $p$ and $q$, or into the condition

$$
(-1)^{\frac{p-1}{2} \frac{q-1}{2}} p^{\frac{q-1}{2}} \equiv+1 \quad(\bmod q) .
$$

However, if the solvability condition $\bmod q$ does not admit integral roots, then

$$
(-1)^{\frac{p-1}{2} \frac{q-1}{2}} p^{\frac{q-1}{2}} \equiv-1 \quad(\bmod q)
$$

holds. By comparison with David Hilbert's Theorem 90 of group cohomology theory ([24], [26], [29]), applied to the quaternionic Galois extension

$$
\mathbb{H}=(1,1)_{\mathbb{R}}=\left(\frac{-1,-1}{\mathbb{R}}\right)
$$

of degree 2, the law of quadratic reciprocity with its supplements follows. In the same vein, the law of quadratic reciprocity is a consequence of the statement that all quadratic fields are contained in cyclotomic fields. More general, the celebrated Kronecker-Weber theorem states that every Abelian field extension of the rational numbers $\mathbb{Q}$ extends to a subfield of the cyclotomic field, so expressible in terms of roots of unity. In other words, a maximal Abelian extension of the field $\mathbb{Q}$ is generated by the torsion points of the action of the ring of integers

$$
\mathbb{Z} \ni m \rightsquigarrow w^{m} \in \mathbb{C}^{\times}
$$

on $w \in \mathbb{C}^{\times}([10],[27])$.

The exponents of the primitive roots modulo $p$ separated within the primitive root power difference of quadratic residues and quadratic non-residues

$$
Y_{1}-Y_{2}=i^{\left(\frac{p-1}{2}\right)^{2}} \sqrt{p}
$$

provides the Gaussian theta series

$$
\left(\frac{p}{q}\right)\left(Y_{1}-Y_{2}\right)=\sum_{0 \leq n \leq q-1}\left(\frac{n}{q}\right) e^{\frac{2 \pi i p}{q} n^{2}}
$$

and the $\operatorname{Mp}(2, \mathbb{R})$ invariant square

$$
\left(Y_{1}-Y_{2}\right)^{2}=(-1)^{\frac{p-1}{2}} p
$$


so that the quadratic pairing $\left(\left(Y_{1}-Y_{2}\right)^{2}, Y_{1}-Y_{2}\right)$ permits to derive the law of quadratic reciprocity by virtue of the identity

$$
i^{\left(\frac{p q-1}{2}\right)^{2}} \sqrt{p q}=\left(\frac{p}{q}\right)\left(\frac{q}{p}\right) i^{\left(\frac{p-1}{2}\right)^{2}+\left(\frac{q-1}{2}\right)^{2}} \sqrt{p} \sqrt{q}
$$

and in accordance with the third order principle of spinor triality. In accordance with the half-spinor Maslov index, the law of quadratic reciprocity implies for the Legendre quadratic symbols

$$
\left(\frac{p}{q}\right)=\left(\frac{q}{p}\right)
$$

if either $p \equiv 1(\bmod 4)$ or $q \equiv 1(\bmod 4)$, and

$$
\left(\frac{p}{q}\right)=-\left(\frac{q}{p}\right)
$$

if both $p \equiv 3(\bmod 4)$ and $q \equiv 3(\bmod 4)$.

The fourth and seventh proofs of Gauß are able to open a route to the cohomological aspect of the law of quadratic reciprocity as indicated earlier in connection with the half-spinor Maslov index which appeared in the context of the two-fold Brauer group $\mathrm{Br}_{2}(\mathbb{R}) \cong \operatorname{Br}(\mathbb{R})$ of order 2. Nevertheless, the half-spinor Maslov index of the metaplectically entangled SchaarLandsberg demonstrates that the central extension $\operatorname{Mp}(2, \mathbb{R})$ of the $\operatorname{symplectic}$ Lie group $\operatorname{Sp}(2, \mathbb{R})$ has nothing lost of its "mysterious" character as a deus ex machina of quantum field theory.

The $\mathbb{R}$-linear independence of the generators of the Brauer group $\operatorname{Br}(\mathbb{R}) \cong \mathbb{Z}_{2}$ gives rise to:

Theorem 3.4. The cyclotomographic procedure of the proof of the law of quadratic reciprocity implements the trivial first cohomologies

$$
\mathrm{H}^{1}\left(\mathbb{C}, \mathbb{Z}_{2}\right)=\{0\}
$$

and correspondingly

$$
\mathrm{H}^{1}\left(\mathbb{C}^{\times}, \mathbb{Z}_{2}\right)=\{1\}
$$
by

It is known that the Hilbert symbol over the local field of $p$-adic numbers $\mathbb{Q}_{p}$ and denoted

$$
\left(\frac{a, b}{p}\right)
$$

is completely determined once its values are known, first for all rational integers $\{a, b\}$ that are prime to $p$, and secondly for all rational integers $a$ that are prime to $p$ with $b=p$. For an odd prime number $p$ and rational integers $\{a, b\}$ prime to $p$, one obtains the equations over $\mathbb{Q}_{p}$

$$
\left(\frac{a, b}{p}\right)=1, \quad\left(\frac{a, p}{p}\right)=\left(\frac{a}{p}\right) .
$$

In the context of global class field theory, the Hilbert symbol determines a duality between the quotient group of the quadratic reciprocity law and itself by means of which it can be identified with its own dual. The Hilbert symbol over 2-adic numbers $\mathbb{Z}_{2} \cong \mathcal{G}$ of rational integers $\{a, b\}$ prime to $p=2$ yields

$$
\left(\frac{a, b}{2}\right)=(-1)^{\frac{(a-1)(b-1)}{4}}, \quad\left(\frac{a, 2}{2}\right)=(-1)^{\frac{a^{2}-1}{8}} .
$$


The Hilbert reciprocity law adopts the product form

$$
\prod_{n}\left(\frac{p, q}{n}\right)=1
$$

where $n$ runs through all prime places including $\infty$ with $\left(\frac{\bullet, \bullet^{\prime}}{\infty}\right)=1$. For any prime number $n$ distinct from $\{p, q, 2\}$, it follows $\left(\frac{p, q}{n}\right)=1$. Hence,

$$
\left(\frac{p, q}{p}\right)\left(\frac{p, q}{q}\right)=\left(\frac{p, q}{2}\right) .
$$

The equation implies the classical law of quadratic reciprocity including the two supplement theorems that are obtained from the product equations

$$
\prod_{n}\left(\frac{-1, p}{n}\right)=1, \quad \prod_{n}\left(\frac{+2, p}{n}\right)=1 .
$$

The duality between the quotient group of the power reciprocity law and itself gives rise to the equivalence of the power reciprocity law and the classical quadratic reciprocity law based on the Abelian field extension by the formalism of local class field theory over the field of rational numbers

$$
\mathbb{Q}\left(\sqrt{(-1)^{\frac{p-1}{2}} p}\right) \mid \mathbb{Q}
$$

and Galois group $\mathcal{G} \cong \mathbb{Z}_{2}$, where $p$ is a positive odd prime ([6], [10], [14], [38]); the field extension of $\mathbb{Q}$ is only ramified at $p>2$. The generalization of the power residue symbol to the Artin symbol and the closely related Hasse's law of reciprocity is regarded as the central result in global class field theory. In the Abelian case, the Artin symbol coincides with the Frobenius symbol.

Theorem 3.5. The classical law of quadratic reciprocity

$$
1=(-1)^{\frac{(p-1)(q-1)}{4}}\left(\frac{p}{q}\right)\left(\frac{q}{p}\right)
$$

follows for any distinct odd primes $p$ and $q \neq 2$ from purely local data.

In his fundamental paper [39], Weil wrote in 1964: Contrary to its appearances, the proof of the law of quadratic reciprocity exposed above does not differ in substance of the classical proof in terms of thet functions and Gaussian sums.

The Artin symbol gives rise to the idélic group epimorphism $J_{\mathbb{Q}} \longrightarrow \mathcal{G}$ with normic kernel in the associated multiplicative idélic vector group $J_{\mathbb{Q}}$ and image of the vector $(1, \ldots, 1, q, 1, \ldots) \in$ $J_{\mathbb{Q}}$, where the $q$ th place is in the component at $p$ th valuation. The image of the vector $(-1,1,1, \ldots) \in$ $J_{\mathbb{Q}}$, where - 1 is in the component at $\infty$, is given by the Legendre symbol

$$
(-1)^{\frac{p-1}{2}}=\left(\frac{-1}{p}\right) \text {. }
$$

For $q=2$, the supplementary identity

$$
1=\left(\frac{2}{p}\right)(-1)^{\frac{p^{2}-1}{8}}
$$

is a consequence of the fact that $(-1)^{\frac{p-1}{2}} p \equiv 1(\bmod 8)$ if and only if $p \equiv \pm 1(\bmod 8)$. 
It is known from the formalism of global class field theory that the locally compact topological group $J_{\mathbb{Q}}$ contains the discrete subgroup $\mathbb{Q}^{\times}$. Algebraically, the idéles of the field of rational numbers $\mathbb{Q}$ can be regarded as a subset of the adéles under the natural injection. As pastings together of local cyclic data, the idéles form the units of the topological ring of adéles $\mathbb{Q}_{\mathbb{A}}$, which is the locally compact adélic ring equipped with the restricted direct product topology and the discrete subring $\mathbb{Q}$ under the diagonal embedding. The duality theory of idéles and adéles endowed with their own restricted direct product topologies represent a natural global extension of the harmonic analysis and Schwartz-Bruhat distribution theory on the adélic ring $\mathbb{Q}_{\mathbb{A}}$ ([38], [11]).

As has been emphasized by Weil in his studies of Siegel's work on quadratic forms, a complicated mathematical theory is not simplified by its specialization, but by neither superficial nor artificial generalizations ([40]). A generalization of this kind is given by the concept of idélization. The idéles are defined in terms of all of the places of a number field $K$ as a finite extension of the field $\mathbb{Q}$. Because the idéles of $K$ carry global information about $K$ in terms of local information about $K$ at each of its places, they are a successful implementation of the local-global principle, which is a recurring theme in quantum field theory and modern number theory, whereby harmonic analysis on the unipotent Heisenberg Lie group $\mathcal{N}$ and its companions have been seen to play an increasingly important role.

\section{CyCLOTOMOGRAPHIC EXTENSIONS}

In his two-part treatise on the theory of biquadratic residues, entitled Theoria residuorum biquadraticorum of 1828, Gauß claims that the theory of quadratic residues had been brought to such a state of perfection that nothing more could be wished. On the other hands, "the theory of cubic and biquadratic residues is by far more difficult ... the previously accepted principles of arithmetic are in no way sufficient for the foundations of a general theory, that rather such a theory necessarily demands that to a certain extent the domain of higher arithmetic needs to be endlessly enlarged ..." In modern language, Gauß is calling for the algebraic theory of spinors and Clifford algebras which culminates in the principle of spinor triality which is due to Élie Cartan (1865-1951). An elegant metaplectically invariant spinor version is due to Claude Chevalley (1909-1984) ([5], [8]).

To derive the law of biquadratic reciprocity in the Euclidean ring $\mathbb{Z}[i] \hookrightarrow \mathbb{C}$ of Gaussian integers with units $\{-1,-i, 1, i\}$ by use of the symplectic machinery, the $(4+1)$-dimensional real unipotent Heisenberg Lie group $\mathcal{N}_{2}$ and real nilpotent Heisenberg Lie algebra $\mathfrak{L} \mathfrak{i e}\left(\mathcal{N}_{2}\right)$ yields the identity

$$
\nu\left(\mathcal{F}_{\mathbb{R} \oplus \mathbb{R}}\right)=J_{4},
$$

where the inclusion

$$
J_{4}=\left(\begin{array}{cc}
0 & -\left(\begin{array}{ll}
1 & 0 \\
0 & 1
\end{array}\right) \\
\left(\begin{array}{ll}
1 & 0 \\
0 & 1
\end{array}\right) & 0
\end{array}\right) \in \operatorname{Sp}(4, \mathbb{R})
$$

holds. If

$$
N: w \rightsquigarrow w \bar{w}=|w|^{2}
$$

denotes the lattice norm in $\mathbb{Z}[i]$, the half-spinor Maslov index -1 of $\operatorname{Mp}(4, \mathbb{R})$ and relatively prime primary elements $\{\rho, \sigma\}$ of $\mathbb{Z}[i]$ give rise to the general law of biquadratic reciprocity, 
which adopts in terms of the quartic Legendre symbols $\left(\doteqdot \bullet^{\prime}\right)_{4}$ the following form

$$
\left(\frac{\rho}{\sigma}\right)_{4}\left(\frac{\sigma}{\rho}\right)_{4}=(-1)^{\frac{N \rho-1}{4} \frac{N \sigma-1}{4}} .
$$

In the context of biquadratic reciprocity, the equation $\left(\frac{\rho}{\sigma}\right)_{4}=1$ holds if and only if the congruence $X^{4} \equiv \rho$ modulo $\sigma$ is solvable in the ring $\mathbb{Z}[i]$ of Gaussian integers, and $\left(\frac{\rho}{\sigma}\right)_{4}=$ $-1,-i$ or $i$ otherwise. Thus

Theorem 4.6. The metaplectic coadjoint orbit model $\mathfrak{L i e}\left(\mathcal{N}_{2}\right)^{*} / \operatorname{CoAd}\left(\mathcal{N}_{2}\right)$ of nilpotent harmonic analysis implements the law of biquadratic reciprocity.

A closely related approach to the law of cubic reciprocity by way of the third order principle of spinor triality proceeds by the ring $\mathbb{Z}[\omega] \hookrightarrow \mathbb{C}$, where

$$
\omega=e^{\frac{2 \pi i}{3}}=\frac{1}{2}(-1+\sqrt{-3}), \quad \bar{\omega}=e^{-\frac{2 \pi i}{3}}=\omega^{2}=\frac{1}{2}(-1-\sqrt{-3})
$$

and $1+\omega+\omega^{2}=0$. The units $\left\{1,-1, \omega,-\omega, \omega^{2},-\omega^{2}\right\}$ of $\mathbb{Z}[\omega]$ form a cyclic group of order 3 . If the rational integer $p \in \mathbb{Z}$ satisfies the congruence $p \equiv 1(\bmod 3)$, then $p=\rho \bar{\rho}$, where $\rho \in \mathbb{Z}[\omega]$ is prime in $\mathbb{Z}[\omega]$. If $p \equiv 2(\bmod 3)$, then $p$ is prime in $\mathbb{Z}[\omega]$. The half-spinor isomorphism of the triality principle can be applied to the residue characters of order three $\left(\bullet \dot{\bullet}^{\prime}\right)_{3}$ or cubic residue characters which play the same role in the theory of cubic residues as the Legendre symbols play in the theory of quadratic residues. The behaviour of cubic residue characters under complex conjugation reads

$$
\overline{\left(\frac{\bullet}{\bullet^{\prime}}\right)_{3}}=\left(\frac{\bar{\bullet}}{\overline{\bullet^{\prime}}}\right)_{3}=\left(\frac{\bullet}{\bullet^{\prime}}\right)_{3}^{2}=\left(\frac{\bullet^{2}}{\bullet^{\prime}}\right)_{3} .
$$

A decomposition of the third power Gaussian sums yields:

Theorem 4.7. For primary prime elements $\left\{\rho_{1}, \rho_{2}\right\} \hookrightarrow \mathbb{Z}[\omega]$ with $N \rho_{1} \neq 3, N \rho_{2} \neq 3$, and $N \rho_{1} \neq$ $\mathrm{N} \rho_{2}$ the order three cubic residue character equation

$$
\left(\frac{\rho_{1}}{\rho_{2}}\right)_{3}=\left(\frac{\rho_{2}}{\rho_{1}}\right)_{3} .
$$

In terms of quantum optics, the cubic residue character equation embodies the quantum entanglement concept of spherical contact geometry.

Remark 4.2. Galois cohomology on the real dual $\mathfrak{L} \mathfrak{i}(\mathcal{N})^{*}$ of the $(2+1)$-dimensional real nilpotent Heisenberg Lie algebra $\mathfrak{L} \mathfrak{i}(\mathcal{N})$ gives rise to an imaginative background of the multiplicative group $\mathrm{H}(\mathbb{R})$ of cyclic factor-classes by the quotient of cyclic factor-sets of degree 2

$$
\frac{\text { covariant factor }- \text { sets }}{\text { coboundary factor }- \text { sets }}
$$

of the groundfield $\mathbb{R}$ ([38], [14]). On smooth differentiable manifolds, the de Rham cohomology is based on the quotient

$$
\frac{\text { closed differential forms }}{\text { exact differential forms }}
$$

to define the de Rham cohomology groups in terms of the coboundaries of closed differential forms. It opens the way to the Kronecker diophantine approximation in number theory via symplectic spinors as symbols of the Fourier integral operators associated with metaplectic mappings ([20], [12]). 
The quaternions represent a quantum field theoretic analogue of the Foucault spherical pendulum device with an open-book foliation of spin echo-stabilized, symplectic swing-planes ([34]). Using a single molecular ion confined in a laser cooled cavity of a linear trap, interferometry experiments permit to ultrasensitively detect the frequency and phase displacement of the pendulum's quantum-enhanced dynamical states which are excited by a sequence of laser-beam driven bichromatic sideband pulses. A calibrated displacement is implemented by exposing the ion to an electric field oscillating at the trapping frequency. The displacement amplitudes of the two-level qubits are in correspondence to the reduced norm of the quaternions.

\section{RATIONAL BIQUADRATIC RECIPROCITY}

It is known that the field of rational numbers $\mathbb{Q}$ has one infinite place corresponding to the embedding

$$
\mathbb{Q} \hookrightarrow \mathbb{Q}_{\infty}=\mathbb{R}
$$

as mentioned supra this place is denoted by $\infty$. The finite places of $\mathbb{Q}$ are in bijective correspondence with the rational primes, with which they will be identified, the place $p$ corresponding to the embedding $\mathbb{Q} \hookrightarrow \mathbb{Q}_{p} \hookrightarrow \mathbb{Q}_{\infty}$. Due to these embeddings, the metaplectic coadjoint orbit model $\mathfrak{L} \mathfrak{i}\left(\mathcal{N}_{2}\right)^{*} / \operatorname{CoAd}\left(\mathcal{N}_{2}\right)$ of the unitary dual $\widehat{\mathcal{N}}_{2}$ of the $(4+1)$-dimensional real unipotent Heisenberg Lie group $\mathcal{N}_{2} \hookrightarrow \mathrm{SL}(5, \mathbb{R})$ is able to derive by means of quantum entanglement the law of rational biquadratic reciprocity. This law answers the following problem: If distinct primes $p \equiv 1(\bmod 4)$ and $q \equiv 1(\bmod 4)$ are given so that $p$ is a fourth power modulo $q$, establish necessary and sufficient conditions that $q$ is a fourth power modulo $p$ ([3]). Actually, the spinor triality principle leads to a number theoretic interpretation of the second Kepplerian law of planetary dynamics through its spherical contact geometry.

The multiplicative group $\mathbb{Z}_{p}^{\times}$admits a unique subgroup of order $\frac{p-1}{4}$ consisting of the residues of fourth powers of integers. Let $\left(\frac{\rho}{p}\right)_{4}$ denote the biquadratic residue character defined by an irreducible $\rho \in \mathbb{Z}[i]$ dividing $p$. Then, $\left(\frac{\rho}{q}\right)_{4}=1$ if and only if the quartic congruence

$$
X^{4} \equiv q \quad(\bmod p)
$$

admits a solution $X \in \mathbb{Z} \hookrightarrow \mathbb{Z}[i]$.

Theorem 5.8. For the quadratic residue character assume $\left(\frac{p}{q}\right)=1$ the law of rational biquadratic reciprocity

$$
\left(\frac{\rho}{q}\right)_{4}\left(\frac{\sigma}{p}\right)_{4}=(-1)^{\frac{q-1}{4}}\left(\frac{p \wedge q}{q}\right)
$$

holds in terms of the Witt invariant -1 of the symbol of norm $\bullet-\bar{\bullet}$ of the four-dimensional involutive central simple $\mathbb{R}$-algebra of quaternions $\mathbb{H} \cong \mathbb{C} \oplus \mathbb{C}$ under quantum entangled cyclotomographic spinor coordinates.

In the context of the spherical contact geometry of the real dual $\mathfrak{L i e}(\mathcal{N})^{*}$, the group isomorphisms

and

$$
\operatorname{Spin}(4, \mathbb{R}) \cong \operatorname{Spin}(3, \mathbb{R}) \times \operatorname{Spin}(3, \mathbb{R})
$$

$$
\operatorname{Spin}(3, \mathbb{R}) \cong \mathrm{SU}(2, \mathbb{C}) \cong \mathrm{SL}(1, \mathbb{H}) \cong \operatorname{Aut}(\mathbb{H})
$$

emphasize the spinor character of the law of rational biquadratic reciprocity. In view of the fact that there is no algebra of quaternions over the field $\mathbb{C}$, the quantum optical phenomenon 
of entanglement is attached to the local field $\mathbb{R}$ and the locally compact connected $\mathbb{R}$-field $\mathbb{H}$ of classical quaternions. The quaternion group $Q_{8}$ with cyclic center $\mathbb{Z}_{8}$ represents a non-Abelian 2-group.

\section{COHERENT THETA DIFFERENTIAL IDÉLES}

Similarly to the theory of zeta-functions of number theory, the metaplectic Schaar-Landsberg construction depends essentially on the concept of Fourier transforms of the metaplectic coadjoint orbit model $\mathfrak{L} \mathfrak{i}(\mathcal{N}) * / \operatorname{CoAd}(\mathcal{N})$ of the unitary dual $\widehat{\mathcal{N}}$ of the $(2+1)$-dimensional real unipotent Heisenberg Lie group $\mathcal{N} \hookrightarrow \mathrm{SL}(3, \mathbb{R})$.

Let $E$ denote a vector space of finite dimension over an $\mathbb{A}$-field $k$ and topological ring $k_{\mathbb{A}}$ of adéles of $k$ in the sense of number theory ([38]). A Haar measure on the tensor product $E_{\mathbb{A}}=E \otimes_{k} k_{\mathbb{A}}$ can be defined by choosing a Haar measure $\alpha_{v}$ on the tensor product $E_{v}=E \otimes_{k} k_{v}$ with symplectic basis $E_{v}^{\circ}$ for each place $v$ so that $\alpha_{v}\left(E_{v}^{\circ}\right)=1$ for almost all $v$. Then, the product measure

$$
\alpha=\prod_{v} \alpha_{v}
$$

and its dual are coherent measures. The measure $\alpha$ on $E_{\mathbb{A}}$ for which $\alpha\left(E_{\mathbb{A}} / E\right)=1$ is known as the Tamagawa measure on $E_{\mathbb{A}}$. Its dual is the Tamagawa measure on $E_{\mathbb{A}}^{*}$.

The field of rational numbers $k=\mathbb{Q}$ admits maximal order $\mathbb{Z}$ and discriminant $1, k_{v}=\mathbb{R}=$ $\mathbb{Q}_{\infty}$ for each place $v, E_{\mathbb{R}}=E \otimes_{\mathbb{Q}} \mathbb{R}$, and the generic flat coadjoint orbit

$$
\mathcal{O}_{1}=E_{\mathbb{R}} \otimes_{\mathbb{R}} \mathbb{C}=E_{\mathbb{C}}
$$

inside the foliation $\mathfrak{L} \mathfrak{i}(\mathcal{N})^{*} / \operatorname{CoAd}(\mathcal{N})$ of the unitary dual $\widehat{\mathcal{N}}$; the one-dimensional center of the real nilpotent Heisenberg Lie algebra $\mathfrak{L} \mathfrak{i} \mathfrak{e}(\mathcal{N})$ is able to parametrize $\widehat{\mathcal{N}}$.

Theorem 6.9. The embedding of the metaplectic coadjoint orbit model $\mathfrak{L} \mathfrak{i}(\mathcal{N}) * / \operatorname{CoAd}(\mathcal{N})$ into the octonionic triality principle of spinor algebra in terms of the coherent dual Tamagawa measure on the dual group $\mathbb{Z} \times \mathbb{Z}$ of the compact two-dimensional torus group $\mathbb{T} \times \mathbb{T}=\mathbb{T}^{2} \hookrightarrow \mathbb{H}$ of the Hopf principal circle bundle with unitary theta characters attached to the rectangular lattice $\Lambda_{1}=\mathbb{Z} \oplus \mathbb{Z} \hookrightarrow \mathrm{WQ}\left(\mathbb{Q}_{2}\right)$ as collection $J_{\mathbb{Q}} \in \mathbb{Q}_{\mathbb{A}}$ of second order differential idéles of module $\left|J_{\mathbb{Q}}\right|_{\mathbb{A}}=1$ on $E_{\mathbb{C}}=\mathcal{O}_{1}$ affords the metaplectic Schaar-Landsberg identity.

The concepts of coherent measure and metaplectic entanglement underline the connection of number theory to the laser realization of spherical contact geometry in the field of quantum optics ([28]).

\section{ENTANGLEMENT OF THE RATIONAL HALF-SPINOR NORM GROUP}

Every rational number is a $p$-adic number, whereby $p$ is a rational prime. A direct calculation of the half-spinor norm with formal power series yields:

Theorem 7.10. The half-spinor norm group $\mathbb{Q}_{2}^{\times} / \mathbb{Q}_{2}^{\times 2}$ which is realized by the law of rational biquadratic reciprocity consists of eight elements represented by $\{1,3,5,7,2,6,10,14\}$.

The ensuing multiplication table for the square classes of $\mathbb{Q}_{2}^{\times}$reads ([32]): 


\begin{tabular}{r||rrrrrrrr} 
& 1 & 3 & 5 & 7 & 2 & 6 & 10 & 14 \\
\hline \hline 1 & 1 & 3 & 5 & 7 & 2 & 6 & 10 & 14 \\
3 & & 1 & 7 & 5 & 6 & 2 & 14 & 10 \\
5 & & & 1 & 3 & 10 & 14 & 2 & 6 \\
7 & & & & 1 & 14 & 10 & 6 & 2 \\
2 & & & & & 1 & 3 & 5 & 7 \\
6 & & & & & & 1 & 7 & 5 \\
10 & & & & & & & 1 & 3 \\
14 & & & & & & & & 1
\end{tabular}

Theorem 7.11. In terms of the Witt quartic group $\mathrm{WQ}\left(\mathbb{Q}_{2}\right) \cong \mathbb{Z}_{2} \times \mathbb{Z}_{8} \times \mathbb{Z}_{2}$, the symmetries of the spinor primciple of triality are represented by the generators

$$
<-1,2>, \quad\langle 1>, \quad\langle-1,5>\text {. }
$$

Since the Witt quartic group $\mathrm{WQ}\left(\mathbb{Q}_{2}\right)$ contains 32 elements, the square class $<1,7>$ of twodimensional quadratic forms over $\mathbb{Q}_{2}$ endows number theoretically the generic flat coadjoint orbit $\mathcal{O}_{1}$ inside the tomographic foliation $\mathfrak{L i e}(\mathcal{N})^{*} / \operatorname{CoAd}(\mathcal{N})$ of quantum field theory with the structure of a hyperbolic plane ([9]).

Corollary 7.2. The action of $\mathbb{Z}_{2} \times \mathbb{Z}_{2} \hookrightarrow \mathrm{WQ}\left(\mathbb{Q}_{2}\right)$ generates the phenomenon of rational quantum entanglement on the two-dimensional hyperbolic space over $\mathcal{O}_{1} \in \mathfrak{L i e}(\mathcal{N})^{*} / \operatorname{CoAd}(\mathcal{N})$ in terms of the Klein four-group $\mathfrak{V}_{4}$.

Thus, the Witt arithmetics admits unexpected applications to the area of quantum field theory.

\section{BY WAY OF CONCLUSION}

When Hermann Weyl (1885-1955), one of the pioneers in introducing non-commutative harmonic analysis of the unipotent Heisenberg Lie group $\mathcal{N}$ into quantum mechanics, wrote his book "The classical groups" in 1939, he overlooked the natural occurrence of the Heisenberg group and the compact, triality conformally triangulated, homogeneous Heisenberg nilmanifold $\Lambda \backslash \mathcal{N}$, exploitation of which opens the gateway to results which one feels Weyl and his former assistant Brauer, the authors of the paper 1935 paper on "Spinors in $n$ Dimensions", would have liked very much. The symbolic calculus of the spherical contact geometry of symplectic spectroscopy at molecular level can be considered as a heritage of Weyl and Brauer ([16], [17]).

Brauer was a former doctoral student of Issai Schur (1875-1911) who supervised his dissertation devoted to the representations of the rotation group by groups of linear transformations. Brauer and Weyl gave a much simpler presentation over Cartan's theory of spinors, based on the close ties with the structures of associative Clifford/Graßmann algebra models. It prepared the portal of the algebraic theory of spinors to the fields of de Rham cohomology theory and supersymmetry ([5], [13]). Brauer's earlier results have opened the way for his definition of the Abelian group of classes of central simple algebras over a commutative groundfield $k$, today called the Brauer group, and denoted by $\operatorname{Br}(k)$ ([38], [32]). Two central simple algebras are said to belong to the same class if the division algebras associated with them by Wedderburn's fundamental theorem are isomorphic. The isomorphy of $\operatorname{Br}(k)$ with the equivalence classes of 
factor-sets over $k$, along with other results of importance for the study of cyclic field extensions such as David Hilbert's Theorem 90, led to the highly elaborate machinery known today as Galois cohomology of local class field theory ([14]). Together with the Stone-von NeumannSegal theorem, the Brauer group $\operatorname{Br}(\mathbb{R})$ and the twofold Brauer group $\mathrm{Br}_{2}(\mathbb{R})$ give rise to an implementation of the basic concepts of quantum entanglement and spin echo, respectively, as outlined in Sections 3 and 7 supra.

A summary of the paper can be given by a quotation of Weyl whose formulation is in his lucid style that only to him was available:

"The problems of Mathematics are not problems in a vacuum. There pulses in them the life of ideas which realize themselves in concreto through our human endeavours in our historical existence, but forming an indissoluble whole transcending any particular science."

The geometric quantization principle mentioned above fits to this philosophy.

\section{REFERENCES}

[1] D.M.S. Bagguley, Editor, Pulsed Magnetic Resonance: NMR, ESR, and Optics. A recognition of E.L. Hahn. Oxford University Press, Oxford, New York, Toronto 1992

[2] O. Baumgart, The Quadratic Reciprocity Law: A Collection of Classical Proofs. Birkhäuser and Springer, Cham, Heidelberg, New York, Dordrecht, London 2015

[3] K. Burde, Ein rationales biquadratisches Reziprozitätsgesetz. J. Reine und Angew. Math. 235, 175-184 (1969)

[4] J.C. Carr, T.J. Carroll, Magnetic Resonance Angiography, Principles and Applications. Springer New York, Dordrecht, Heidelberg, London 2012

[5] C. Chevalley, The Algebraic Theory of Spinors and Clifford Algebras. Collected Works, Volume 2, SpringerVerlag, Berlin, Heidelberg, New York 1997

[6] N. Childress, Class Field Theory. Springer-Verlag, Berlin, Heidelberg, New York 2009

[7] C.W. Curtis, Pioneers of Representation Theory: Frobenius, Burnside, Schur, and Brauer. American Mathematical Society, Providence, Rhode Island 1999

[8] J.A. Dieudonné, Review of The Algebraic Theory of Spinors by C. Chevalley. Bull. Amer. Math. Soc. 60, 408-413 (1954)

[9] J. Franchi, Y. Le Jan, Hyperbolic Dynamics and Brownian Motion: An Introduction. Oxford University Press, Oxford, New York 2012

[10] L.J. Goldstein, Analytic Number Theory. Prentice Hall, Englewood Cliffs, New Jersey 1971

[11] G. Gras, Class Field Theory: From Theory to Practice. Springer-Verlag, Berlin, Heidelberg, NewYork 2005

[12] V.W. Guillemin, Symplectic spinors and partial differential equations. In: Géomeétrie Symplectique et Physique Mathématique, pp. 217-252, Centre National de la Recherche Scientifique, Paris 1975

[13] V.W. Guillemin, S. Sternberg, Supersymmetry and Equivariant de Rham Theory. Springer-Verlag, Berlin, Heidelberg, New York 1999

[14] D. Harari, Galois Cohomology and Class Field Theory. Springer Nature, Cham, Switzerland 2020

[15] R. Howe, On the role of the Heisenberg group in harmonic analysis. Bull. Amer. Math. Soc. (New Series), Vol. 3, 821-843 (1980)

[16] R. Howe, Quantum mechanics and partial differential equations. J. Funct. Anal. 38, 188-254 (1980)

[17] R. Howe, The oscillator semigroup. In: The Mathematical Heritage of Hermann Weyl. R.O. Wells, Jr., Editor, Proceedings of Symposia in Pure Mathematics, Vol. 48, pp. 61-132, American Mathematical Society, Providence, Rhode Island 1988

[18] H. Johansen-Berg, T.E.J. Behrens, Editors, Diffusion MRI: From Quantitative Measurement to In vivo Neuroanatomy. Elsevier, Academic Press, Amsterdam, Boston, Heidelberg 2009

[19] D.K. Jones, Editor, Diffusion MRI: Theory, Methods, and Applications. Oxford University Press, Oxford, New York 2010

[20] B. Kostant, Symplectic spinors. In: Istituto Nazionale di Alta Matematica Roma, Symposia Mathematica, Volume XIV, pp. 139-152, Academic Press, London, New York 1974

[21] S. Lang, Introduction to Algebraic and Abelian Functions. Second edition, Springer-Verlag, New York, Heidelberg, Berlin 1987

[22] G. Lion, M. Vergne, The Weil Representation, Maslov Index and Theta Series. Birkhäuser Verlag, Boston, Basel, Stuttgart 1980

[23] A. Merkurjev, On the norm residue symbol of degree 2. Sovjet Math. Doklady 24, 546-551 (1981)

[24] P. Morandi, Field and Galois Theory. Springer-Verlag, New York, Berlin, Heidelberg 1996 
[25] S. Mori, Introduction to Diffusion Tensor Imaging. Elsevier, Amsterdam, Boston, Heidelberg 2007

[26] J. Neukirch, Class Field Theory. Springer-Verlag, Berlin, Heidelberg, New York 1986

[27] O.T. O'Meara, Introduction to Quadratic Forms. Springer-Verlag, Berlin, Heidelberg, NewYork 2000

[28] A.M. Ozorio de Almeida, Entanglement in phase space. In: Entanglement and Decoherence: Foundations and Modern Trends, A. Buchleitner, C. Viviescas, M. Tiersch, Editors, Lecture Notes in Physics 768, pp. 157-219, Springer-Verlag, Berlin, Heidelberg, New York 2010

[29] R.S. Pierce, Associative Algebras. Springer-Verlag, New York, Heidelberg, Berlin 1982

[30] C.R. Riehm, Introduction to Orthogonal, Symplectic and Unitary Representations of Finite Groups. American Mathematical Society, Providence, Rhode Island 2011

[31] H. Salzmann, D. Betten, T. Grundhöfer, H. Hähl, R. Löwen, M. Stroppel, Compact Projective Planes, With an Introduction to Octonion Geometry. Walter de Gruyter, Berlin, New York 1995

[32] W. Scharlau, Quadratic and Hermitian Forms. Springer-Verlag, Berlin, Heidelberg, New York 1985

[33] W.J. Schempp, Magnetic Resonance Imaging: Mathematical Foundations and Applications. Wiley-Liss, New York, Chichester, Weinheim 1998

[34] W.J. Schempp, Dynamic metaplectic spinor quantization: The projective correspondence for spectral dual pairs. Journal of Applied Mathematics and Computing 59, 545-584 (2019)

[35] W.J. Schempp, Applications of metaplectic cohomology and global-local contact holonomy. Journal of Applied Mathematics and Computing 67 (2), 2021

[36] E.O. Stejskal, Use of spin echoes in a pulsed magnetic-field gradient to study non-isotropic, restricted diffusion and flow. J. Chem. Phys. 43, 3597-3603 (1965)

[37] E.O. Stejskal, J.E. Tanner, Spin diffusion measurements: Spin echoes in the presence of a time-dependent field gradient. J. Chem. Phys. 42, 288-292 (1965)

[38] A. Weil, Basic Number Theory. Third edition, Springer-Verlag, Berlin, Heidelberg, New York 1974

[39] A. Weil, Sur certains groupes d'opérateurs unitaires. Acta Math. 111, 143-211 (1964); Collected Papers, Vol. III, pp. 1-69, Springer-Verlag, New York, Heidelberg, Berlin 1979

[40] A. Weil, Sur la formule de Siegel dans la théorie des groupes classiques. Acta Math. 113, 1-87 (1965); Collected Papers, Vol. III, pp. 71-157, Springer-Verlag, New York, Heidelberg, Berlin 1979

WALTER J. SCHEMPP

UNIVERSITÄT SIEGEN

LEHRSTUHL FÜR MATHEMATIK I

57068 SIEGEN, GERMANY

ORCID: 0000-0001-6199-5863

E-mail address: schempp@mathematik . uni-siegen.de 